HNO 2010 $58: 5$

DOI 10.1007/s00106-009-2049-2

๑) Springer Medizin Verlag 2009

\section{Dank an die Gutachter}

Die HNO legt alle eingesandten Manuskripte wenigstens zwei Fachgutachtern zur Begutachtung vor. Die Zahl derer, die uns hierbei im Jahre 2009 unterstützt haben, ist groß. Unsere Gutachter kommen aus der Hals-Nasen-Ohrenheilkunde mit all ihren Teilgebieten. Einen bedeutenden Anteil am Begutachtungsprozess haben jedoch auch Naturwissenschaftler unterschiedlicher Fachrichtungen und vor allem viele Kolleginnen und Kollegen aus den klinischen Nachbardisziplinen, die ihren Sachverstand bereitwillig eingebracht haben. Wir möchten daher die erste Ausgabe des neuen Jahres nutzen, um all jenen unseren herzlichen Dank auszusprechen, die durch ihre Gutachtertätigkeit dazu beigetragen haben, diese Zeitschrift zu einem lebendigen Forum des wissenschaftlichen Austausches und der Fortbildung zu machen.

\author{
Aschendorff, Freiburg \\ Bachert, Gent \\ Bankfalvi, Essen \\ Bas, Düsseldorf \\ Baumann, I., Heidelberg \\ Baumann, U., Frankfurt \\ Berger, Marburg \\ Berghaus, München \\ Betz, München \\ Beutner, Köln \\ Bless, Tübingen \\ Bloching, Berlin \\ Bootz, Bonn \\ Böschke, Göttingen \\ Brand, Oldenburg \\ Briner, Zürich \\ Bruchhage, Augsburg \\ Brunner, Augsburg \\ Buecheler, Bonn \\ Cornelius, Dortmund \\ Dazert, Bochum \\ Delb, Homburg \\ Dellian, München \\ Deuster, Heilbronn \\ Dietz, Leipzig \\ Dyckhoff, Heidelberg \\ Eckel, Klagenfurt \\ Ehrt, Rostock \\ Ernst, Berlin \\ Fauser, Hamburg \\ Federspil, Heidelberg \\ Flatz, Innsbruck \\ Gerstner, Bonn \\ Gissmann, Heidelberg \\ Grabein, Augsburg \\ Gross, Berlin \\ Gubisch, Stuttgart \\ Haberkorn, Heidelberg \\ Hagen, Würzburg \\ Harreus, München \\ Häusler, Bern
}

Haxel, Mainz

Heiden, Traunstein

Helbig, M., Frankfurt

Helbig, S., Frankfurt

Hempel, Wiesbaden

Heppt, Karlsruhe

Herzog, Leipzig

Hess, Hamburg

Hesse, Bad Arolsen

Hoffmann, Kiel

Holtmannspoetter, Münster

Hörmann, Mannheim

Ihrler, München

Iro, Erlangen

Jakob, Bonn

Kiening, Augsburg

Kiese-Himmel, Göttingen

Kleinsasser, Würzburg

Klingmann, Heidelberg

Klussmann, Köln

Knecht, Hamburg

Knipper, Tübingen

Koch, Erlangen

Koitschev, Tübingen

Kramer, München

Krause, München

Kummer, München

Lamprecht, Essen

Landerl, Salzburg

Lang, Essen

Laszig, Freiburg

Lenarz, Hannover

Lesinski-Schiedat, Hannover

Leunig, München

Liebler, Heidelberg

Lorenz, UIm

Löwenheim, Tübingen

Luckhaupt, Dortmund

Mast, München

Maune, Köln

Maurer, Koblenz
Mazurek, Berlin

Meister, Köln

Meyer, H. Salzburg

Meyer, J.E., Lübeck

Mösges, Köln

Münscher, Hamburg

Naumann, Homburg

Nawka, Berlin

Nawroth, Heidelberg

Nickisch, München

Olzowsky, München

Ostertag, Kufstein

Peraud, München

Pfister, Tübingen

Pitten, Gießen

Plontke, Tübingen

Plotz, Oldenburg

Praetorius, Heidelberg

Probst, Zürich

Przybilla, München

Ptok, Hannover

Quante, Köln

Quetz, Kiel

Rachinger, München

Raspe, Lübeck

Ratzlaff, Heidelberg

Regauer, Graz

Reichel, München

Reith, Saarbrücken

Riechelmann, Innsbruck

Rosanowski, Erlangen

Rotter, UIm

Rudack, Kiel

Saloga, Mainz

Schäfer, Bochum

Schaller, München

Scheithauer, München

Schipper, Düsseldorf

Schlemmer, München

Schneider, Würzburg

Schöler, Heidelberg
Schönweiler, Lübeck

Schröder, Lübeck

Schuster, Erlangen

Schwarting, Mainz

Schwarz, Erlangen

Simmen, Luzern

Sommer, Osnabrück

Stammberger, Graz

Steffens, Regensburg

Strauß, Leipzig

Strieth, München

Stuck, Mannheim

Stuhrmann, Heidelberg

Sudhoff, Bielefeld

Tasman, St. Gallen

Tesche, Hamburg

Todt, Berlin

von Koch, München

Walger, Köln

Wallner, Heidelberg

Welge-Lüssen, Basel

Werner, Marburg

Wiederhöfer, Heidelberg

Windfuhr, Duisburg

Wittekindt, Jena

Wollenberg, Lübeck

Zahnert, Dresden

Zenk, Erlangen 